\title{
Dal Teatro di Euripide Al Cinema di Pasolini: LA Mise eN ABIME Del Mito di Medea
}

W Alter Zidaric ${ }^{1}$

ABSTRACT: Il mito di Medea continua a affascinare l'umanità, fin dall'antichità, soprattutto nella versione di Euripide. Si commentano composizioni artistiche che riprendono im mito di Medea e si analizza, in particolare, il rapporto Medea, Callas, Pasolini.

PAROLE CHIAVE: Medea; Euripide; Cherubini; mito; Callas; Pasolini; opera; cinema. 
RESUMO: O mito de Medéia exerce seu fascínio sobre a humanidade desde a antiguidade, especialmente na visão de Eurípides. São comentadas algumas composições artísticas que retomam o mito de Medéia e, em particular, analisa-se a relação Medéia, Callas, Pasolini.

PALAVRAS-CHAVE: Medéia; Eurípides; Cherubini; mito; Callas; Pasolini; ópera; cinema.

ABSTRACT: Medea myth has fascinated humanity ever since antiquity, especially on Euripides point of view. Some artistic compositions which refer to Medea myth are commented, we especially analyze Medea, Callas and Pasolini relation.

KEYWORDS: Medea; Euripides; Cherubini; myth; Callas; Pasolini; opera; cinema. 
Il mito è una menzogna che dice sempre la verità.

Jean Cocteau

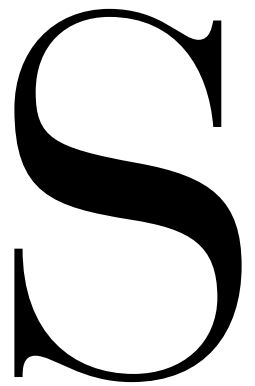

in dall'antichità e con Euripide in particolare, che ha cristallizzato una delle versioni del mito che ha finito coll'imporsi nell'immaginario collettivo attraverso i secoli, Medea - il cui mito è attestato all'epoca micenea, all'inizio della seconda metà del secondo millennio - non ha smesso di affascinare l'umanità. Gli artisti - scrittori, scultori, pittori, drammaturghi, musicisti, cineasti, coreografi - se ne sono ispirati nei campi più svariati e in tutte le epoche, fino alla nostra. In effetti, l'ultima composizione ad aver tratto ispirazione dalla maga infanticida è alquanto recente: si tratta del balletto Le Songe de Médée (Il sogno di Medea) del coreografo Angelin Preljocaj, su una musica dell'italiano Mauro Lanza (nato nel 1975), rappresentato al Palais Garnier di Parigi nel novembre 2004. Con questa terza creazione per il Balletto dell'Opéra National di Parigi, Preljocaj si è appropriato a sua volta questo personaggio complesso e nel programma di sala si può leggere che "l'antica Medea interessa il coreografo altrettanto quanto quelle che sono protagoniste di 'fatti di cronaca attuali' . Le Songe de Médée gli consente di evocare ciò che potrebbe essere il desiderio nas- 
costo, l'inconscio dell'eroina, a monte del mito"2. Prima di questo balletto, parecchie composizioni, sia musicali che teatrali, si sono interessate a tale soggetto negli ultimi anni come, ad esempio:

le opere

- Medeamaterial (1990), di Pascal Dusapin su un libretto in tedesco di Heiner Müller, rappresentata il 13 marzo 1992 al Teatro de la Monnaie a Bruxelles;

- Medea, di Gordon Kerry su un libretto in inglese e in latino di Justin Macdonnell tratto da Seneca, rappresentata nel 1993 a Melbourne;

- Medea (1998), di Rolf Liebermann su un libretto in tedesco di Ursula Hass, rappresentata il 12 febbraio 2002 all'Opéra di Parigi (in francese);

- Midea (1999) di Oscar Strasnoy su un libretto in italiano di Irina Possamai, rappresentata l' 8 settembre 2000 al Teatro Caio Melisso di Spoleto ("una riscrittura del mito di Medea"');

- Medea in Korinth (2002) oratorio di Georg Katzer in tedesco;

- Médée, di Michèle Reverdy, su un libretto in francese di Kai Stefan Fritsch e Bernard Banoun, tratto dal romanzo di Christa Wolf, rappresentata all'Opéra di Lione il 25 gennaio 2003;

i drammi teatrali

- Médée (1992), di Max Rouquette (tradotto dall'occitano in francese dall'autore);

- Manhattan Medea (1999), di Dea Loher, in tedesco;

- Matériau-Médée (2001), tradotto da Heiner Müller, rappresentato a Bruxelles;

- Médée Kali (2003), dramma in francese di Laurent Gaudé;

- L'Effet Médée (2003), dramma in francese di Suzie Bastien, rappresentato a Montréal nel marzo 2005.

Si tratta dunque di un numero assai importante di lavori a partire dallo stesso mito e per di più concentrati in un periodo storico molto breve, in realtà, il che costituisce un fatto sorprendente e singolare su cui tornerò nella mia conclusione.

2. Preljocaj assegna, inoltre, un ruolo molto particolare ai due bambini che intervengono nel dramma giacché, come ha affermato nell'ottobre 2004: "era l'occasione di mettere in scena i bambini, non come dei 'fantocci', facendoli apparire soltanto all'inizio e nel momento della loro morte, ma per mettere in risalto la relazione madre/figli in modo molto più acuto e sensuale. Assegnare loro un vero ruolo significa rendere ancora più forte il contrasto tra l'amore di una madre e la violenza dell'infanticidio". Le citazioni del coreografo sono tratte dal Programma della stagione 2004-2005 dell'Opéra National di Parigi. Tutte le traduzioni sono mie qualora non compaia il nome del traduttore. 


\section{Euripide (480-406 A. C.)}

Una delle grandi novità del teatro di Euripide è che "il personaggio assume una tale dimensione emotiva da sconcertare, l'amore diventa un tema essenziale che ha come elemento motore la donna. Più dei suoi predecessori, egli vuole svelare il misterioso femminino: l'istinto materno, la passione, il furore e la gelosia"4. Personaggio estremamente complesso, crogiuolo di interrogativi violenti e incessanti, tra ragione e passione, la Medea di Euripide, a causa del gesto finale dell'infanticidio, di cui non c'è traccia nelle versioni precedenti (almeno otto) della leggenda, si impone in tutta la sua dimensione assolutamente tragica e mostruosa raggiungendo il sublime ${ }^{5}$, nel senso romantico del termine, mentre Giasone, eroe eminentemente positivo nelle prime versioni del mito, aiutato dagli dei, è trasformato in un semplice codardo. Medea la maga, la barbara venuta da altri lidi, da un mondo senz'altro primitivo, è un'esiliata, una sradicata, in ultimo ripudiata e costretta a un nuovo esilio in una Grecia che è pur tuttavia la culla della cultura e della civiltà mediterranea, in qualche modo simbolo del mondo moderno e civilizzato: "io sola sono, senza patria, e oltraggio / mio marito mi fa, che me rapiva / da una barbara terra; e non ho madre, / non fratello o parente, a

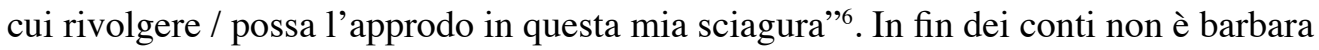
colei che sembra esserlo di più, poiché dietro questi interrogativi lancinanti di Medea si tratta proprio di una rimessa in discussione dei rapporti umani pubblici e privati in un'epoca in cui Atene attraversa una crisi profonda e non domina più le altre città greche. In Euripide si è catapultati in medias res, già a Corinto, gli antefatti della storia di Medea e Giasone sono stati raccontati dalla balia e si assiste direttamente allo scioglimento della tragedia.

\section{Luigi Cherubini (1760-1842)}

Il mito greco di Medea alimenta sin dal $16^{\circ}$ secolo l'arte europea ${ }^{7}$ e, tra le varie realizzazioni artistiche, una di quelle che ha finito per imporsi alla fine del 700 è di ordine teatral-musicale: si tratta dell'opera di Luigi Cherubini, "tragedia in tre atti",

3. Possamai, I. Midea (libretto), Milano, Casa Ricordi, 2003, p. 69.

4. Landrain, M. «Préface: La Médée d'Euripide», in: EuriPide, Médée (suivi de Les Troyennes), trad. dal greco antico di V.-H. Debidour, Paris, Librio 527, (1999) 2002, p. 7.

5. "Il poeta aveva per abitudine di rinnovare le leggende inventando loro uno scioglimento imprevisto e prendendo spesso in contropiede i suoi detrattori. Sceglieva la versione rara di un mito e prendeva il rischio di allontanarsene" (Ibidem, p. 8).

6. EuriPIDE, Medea, trad. di Ettore Romagnoli, in: <http://www.rodoni.ch/busoni/euripide/euripidemedea42.htm>, consultato il $18 / 8 / 2010$.

7. È molto difficile stilare una lista esauriente delle opere ispiratesi al mito di Medea proprio a causa del loro gran numero e dei generi diversi, visto che interessano tutti i campi artistici, tuttavia il sito <http://fr.wikipedia.org/wiki/Médée>, fornisce una lista interessante di 
su un libretto di François-Benoît Hoffman fedelmente ispiratosi alla tragedia omonima di Corneille (che a sua volta era partito da Euripide). Médée di Cherubini, nella versione originale francese di opéra-comique è rappresentata al Teatro Feydeau di Parigi, il 23 ventoso (13 marzo) 1797, in pieno periodo rivoluzionario, e come scrisse il Courrier des spectacles dell'indomani della prima: "La tragedia lirica di Medea ha ottenuto il successo più brillante e più meritato. La musica, per la quale gli elogi non sono mai troppi, è di Cherubini [...] le scene sono magnifiche, infine nulla è mancato a quest'opera per renderla degna della massima ammirazione"s.

La versione italiana dell'opera si impose in Europa dalla seconda metà dell' 800 , per esempio a Londra nel 1865, con recitativi di Luigi Arditi sostituiti ai dialoghi originari, ma Medea fu rappresentata in Italia, alla Scala di Milano, soltanto il 30 dicembre 1909, nella versione ritmica italiana di Carlo Zangarini, con Ester Mazzoleni, diva dell'epoca, nel ruolo eponimo. Malgrado l'attesa, l'accoglienza del pubblico e della critica fu tiepida e tra le recensioni una in particolare, apparsa sul Corriere della Sera, e relativa all'interpretazione della primadonna, assunse accenti profetici:

[...] Ed ecco Medea. Il suo apparire improvviso, il terrore che ella inspira, avvolta nei neri suoi veli, pallida, fremente di sdegno e di amore, non commuovono l'orchestra ma l'effetto non manca. La Mazzoleni campeggia sul fondo chiaro della scena in un atteggiamento e un gesto veramente tragici. Il pubblico già l'ammira. [...] Ma, come ella incomincia il suo canto, non la riconosce non gli sembra più la stessa. La bella voce dolce, fluida, squillante, non è più sì pura e sì intensa. La parte di Medea appare subito troppo faticosa per la squisita artista, parte troppo grave per qualunque delle odierne cantatrici. Chi possiede più una voce sì robusta $\mathrm{e}$ sì piena, sì facile $\mathrm{e}$ sì intensa da reggere allo sforzo di un canto continuamente concitato, esteso dalle note più gravi alle più acute, che deve essere accompagnato da un'azione scenica delle più violente e delle più espressive? La Mazzoleni ha cantato ieri con grande efficacia d'accento, con una chiara pronuncia, con un senso drammatico ammirevole; ma non poté dare alla sua voce tutta la violenza della passione di Medea, l'urlo dell'angoscia e della ferocia. E forse anche per questo la tragedia musicale non riuscì ad afferrare il pubblico, a scuoterlo, ad avvincerlo alla sua vicenda, ad atterrirlo colla sua catastrofe $[\ldots]^{9}$.

nomi di artisti che hanno rappresentato il mito a partire dal $16^{\circ}$ secolo.

8. Giommarini, G. "La nascita dell'opera”. In: Cherubini, L. Medea, dir. N. Rescigno, orchestra Royal Opera House Covent Garden, Londra 1959, 2 CD, Allegato al fascicolo n 10 di Opera Italiana, Armando Curcio editore, 1990, p. 29. L'opera di Cherubini ebbe una vita teatrale piuttosto breve in Francia; in compenso, fu molto popolare nei paesi di lingua tedesca e molto apprezzata da musicisti quali Beethoven, Wagner e Brahms.

9. Citato in Ibidem, nel libretto che accompagna il CD Medea, pp. 32-34 (“Medea in Italia da Mazzoleni a Callas”). 
Medea di Cherubini scompare dal repertorio dopo questa fugace apparizione e torna alla ribalta soltanto circa cinquant'anni dopo, il 7 maggio 1953, quando viene rappresentata al Teatro Comunale di Firenze durante il Maggio Musicale fiorentino, con la direzione di Vittorio Gui e con l'interpretazione di Maria Callas nel ruolo eponimo. Sia il pubblico che la critica gridarono al miracolo e persino un critico e musicologo non callasiano, come il Celli, scrisse sul Corriere Lombardo dell'8 maggio 1953: "Una meraviglia. Una grande cantante e un'attrice tragica di impressionante potenza. Ella ha dato alla maga una vocalità torva, ferocemente intensa nel registro grave, terribilmente squillante nell'acuto" 10 . Medea diventa uno dei cavalli di battaglia del soprano che tra il 1953 e il 1962 canta il ruolo un po' ovunque per il mondo (Milano, Dallas, Londra, Epidauro, ecc.). Come la critica ha più volte ribadito, Maria Callas è stata LA grande interprete di Medea di Cherubini: è stata lei a riscoprire e a reinventare il ruolo ${ }^{11}$.

Tra il 1962 e il 1965 le apparizioni di Maria Callas sulla scena si fanno sempre più rare: l'ultima rappresentazione cui prende parte è quella della Tosca londinese, che si svolge il 5 luglio 1965. Poi è il silenzio assoluto. Parallelamente al declino vocale che accompagna quegli anni e che influisce sulla sua vita pubblica di diva internazionale, sul versante della vita privata le cose non vanno affatto meglio e la sua relazione con Onassis, che dura dal 1959, non si concretizza come ella vorrebbe e come il celebre uomo d'affari le fa sperare. Infine, il 20 ottobre 1968, Onassis sposa Jackie Kennedy creando la sorpresa generale e a insaputa di Maria Callas, la sua compagna da quasi un decennio, che incassa il colpo in stato di shock. Ecco dunque aprirsi per lei un periodo molto difficile e doloroso che ella tenta di fronteggiare moltiplicando le uscite mondane e sfoggiando un perfetto fair play di facciata ${ }^{12}$. La sola e unica salvezza poteva venire soltanto dal lavoro: un nuovo progetto, un nuovo ruolo... Già da qualche anno il cinema la corteggiava ricevendo, però, ogni volta un rifiuto da parte sua. Tuttavia, sono tre le proposte che sembrano inizialmente stimolare il suo interesse: un film sulla vita di Giacomo Puccini, progetto che Luchino Visconti accarezzava da tempo, l'opera The Consul di Gian Carlo Menotti, che il compositore italo-americano aveva pensato di offrirle già all'epoca della prima, nel 1950, e un film di Joseph Losey ispirato al dramma Boom! di Tennessee Williams. Tuttavia, sarà un'altra proposta che le viene in un secondo tempo da Visconti ad attrarla irresistibilmente: una nuova produzione

10. Ibid., p. 34

11. Seguendo le tracce della Callas, alcune artiste si sono cimentate nel ruolo di Medea: nelle incisioni discografiche, per esempio, Rita Gorr, Gwyneth Jones e Sylvia Sass; sulla scena: Magda Olivero (1967-1968, 1970-1971), Leonie Rysanek (tra il 1972 e il 1976), Montserrat Caballé (1976), Katia Ricciarelli (1985), Shirley Verret (1986) e, di recente, Anna Caterina Antonacci (2005).

12. La Callas ha registrato la sua testimonianza su quegli anni, su quel periodo doloroso con John Ardoin, il grande critico musicale americano, uno dei suoi amici più fedeli con cui si confidava regolarmente. 
della Traviata all'Opéra di Parigi, messa in scena da lui stesso, e per la quale firmano addirittura il contratto con il celebre teatro. Ecco, però, che la paura di un fiasco in una produzione che avrebbe attirato l'attenzione del mondo intero finisce col diventare una minaccia per la Callas la quale esige, allora, dalle venti alle trenta prove con coro e orchestra perfettamente consapevole del fatto che la sua richiesta non potrà essere soddisfatta.

\section{Maria Callas (1923-1977) e Pier Paolo Pasolini (1922-1975)}

Accentando infine la proposta per un film di Pier Paolo Pasolini, Medea, sarà lei questa volta a creare la sorpresa generale.

In un'intervista ricordò il giorno esatto della proposta: 19 ottobre 1968. Facendo buon viso alla difficile decisione, disse: "Un anno dopo, Rossellini venne da me con un progetto concreto di Pasolini [...] e ci incontrammo in una felice coincidenza di volontà" ${ }^{13}$.

Occorre aprire qui una parentesi: più di un anno dopo la proposta di Franco Rossellini, produttore del film, la Callas afferma dunque in maniera alquanto singolare che quest'ultima le sarebbe stata fatta il 19 ottobre 1968, cioè alla vigilia del matrimonio di Onassis con Jackie Kennedy! È molto difficile crederle su questo punto e tale coincidenza non è certamente fortuita. In effetti, agendo così, ella ha senz'altro voluto dimostrare di aver preso la decisione di fare il film prima della notizia ufficiale del matrimonio del celebre armatore, in maniera quindi del tutto indipendente, ma si tratterebbe, stando a quanto afferma, soltanto di un lasso di tempo di ventiquattrore. Ciò appare allora del tutto inverosimile dato lo stato di profonda depressione in cui versava in quel momento. La scelta del film di Pasolini le si prospetta piuttosto come una possibilità, cadutale dal cielo, per dimenticare e, al contempo, affermarsi. Nel caso in cui il film avesse avuto successo, ciò avrebbe potuto significare per lei l'inizio di una nuova carriera; in ogni modo, si trattava davvero di ritornare a lavorare, cosa che la Callas, da grande artista professionista, apprezzava enormemente: "Parce que

13. Citazione tratta da: <http://www.pasolini.net/callasPPP_medea-Schwartz.htm>, (SCHWARTZ, B. D. Pasolini Requiem, trad. it. di Paolo Barlera, Venezia, Marsilio, 1995). L'intervista in questione fu fatta alla Callas da Giacomo Gambetti a Cinecittà, alla metà del dicembre 1969. 
j'aime travailler; travailler, c'est toujours bien, c'est comme cela que je m'amuse vraiment" ${ }^{14}$. Il lavoro era però anche $\mathrm{e}$, forse, soprattutto un diversivo indispensabile, una maniera di non pensare alla situazione tragica e dolorosa in cui si trovava ${ }^{15}$. Maria Callas accetta senza alcun dubbio di impersonare Medea sullo schermo anche per un'altra ragione: la mise en abyme della sua storia d'amore con Onassis che è appena finita in modo brutale e che l'ha fatta sprofondare nella disperazione. Decide quindi di reagire vendicandosi simbolicamente dell'uomo che l'ha tradita: Onassis/Giasone. Durante le riprese del film, la Callas dichiara alla stampa a proposito del personaggio di Medea:

Era una creatura semidivina che aveva riposto tutta la sua fiducia in un uomo. Ma al tempo stesso era una donna ricca di tutte le esperienze femminili: esperienze che comportavano dolori, sacrifici. Medea è passata attraverso tutto ciò, sforzandosi di sopravvivere. È difficile spiegarlo a parole... ho cominciato a guardare nel profondo della sua anima ${ }^{16}$.

Certo, quando parla di "tutte le esperienze femminili" le si potrebbe facilmente obiettare che non è mai stata madre... e si prenderebbe un grosso granchio come ha dimostrato Nicholas Gage giacché, nel segreto più assoluto, la Callas aveva dato alla luce un bambino, il 30 marzo 1960, che era poi morto qualche ora dopo il parto ${ }^{17}$.

Laura Betti, amica intima e musa di Pasolini, sconsiglia il cineasta quando egli sceglie la Callas: lo dice testualmente in un'intervista trasmessa dal canale televisivo ARTE nel 1997, durante una serata commemorativa per i vent'anni della scomparsa della cantante. Ella è categorica, anche per gelosia personale, sul fatto che la Callas non potrà essere una buona Medea, tuttavia Pasolini non demorde e nell'intervista Laura Betti finisce col lanciare un'affermazione che mi consente di avvalorare il mio proposito: "Callas era un mito!"18.

$\mathrm{Se}$, riportando poco fa le parole della Callas, abbiamo potuto capire le ragioni della scelta riguardo Pasolini - affascinata, in realtà, dall'intelligenza e dagli artisti colti e raffinati, si infatua del poeta cineasta ${ }^{19}$ così come si era infatuata di Luchino Visconti qualche anno prima - perché, invece, quest'ultimo finisce con lo scegliere lei? Ecco quanto afferma il cineasta durante le riprese del film: "Siamo di fronte ad

14. Ibidem, p. 20.

15. Fino a che punto Pasolini e i produttori del film non hanno colto l'occasione dell'enorme scandalo sulla stampa internazionale riguardo alla rottura tra la Callas e Onassis e al matrimonio di quest'ultimo con la vedova Kennedy per uno scoop pubblicitario possibile per il loro film? Come afferma Gage, "naturalmente il regista sapeva bene che il pubblico avrebbe fatto dei paralleli tra la passione omicida di Medea per Giasone, che l'abbandona per sposare la figlia di un re, e la nota, sfortunata, relazione di Maria con Onassis" (GAGE, N. Fuoco greco. La storia di Maria Callas e Aristotele Onassis, trad. di G.M. Giughese, Milano, Sperling \& Kupfer, 20

16. Citato in Arianna Stassinopoulos, Maria Callas al di là della leggenda, trad. dall'inglese di R. Maindardi, Milano, A. Vallardi (Garzanti editore), 1982, pp. 341-342. 
un'artista che in un certo senso è la più moderna delle donne. Tuttavia vive in lei un creatura antica, strana, misteriosa, arcana che nasconde terribili conflitti interiori”" ${ }^{20}$. Ora è difficile immaginare che una tale definizione possa applicarsi in modo indiscriminato a tutti gli artisti e credo che egli colga qui l'essenza della donna Callas, proprio il misterioso femminino, e nondimeno aggiunge: "Sono perfettamente conscio delle sue capacità professionali, ma per me contano poco. Sono state le sue peculiarità umane a farmi capire che la Callas poteva essere la mia Medea"21.

Quando parla di creatura antica, Pasolini fa senza dubbio allusione anche al fatto che la Callas, la quale è greca, non dimentichiamolo, diventa IL simbolo ai suoi occhi di quel mondo e di quella civiltà antica che, nonostante tutto, traspaiono dalla sua persona oltre il lato mondano del personaggio. Ma la Callas era già un vero e proprio mito all'epoca delle riprese del film ${ }^{22}$, come Laura Betti ricorda nell'intervista sul canale televisivo ARTE, donde l'interesse tanto estetico quanto ideologico del procedimento pasoliniano di utilizzarla in maniera originale rispetto alla sua carriera di cantante d'opera, cioè di "umanizzarla" in qualche modo, privandola di quella "voce" che aveva fatto di lei una diva, una dea adulata e mitizzata dalle folle, per mostrarla invece in quanto donna tout court, banalmente sedotta e abbandonata. Tutto ciò, inoltre, in un film che nasce come un puro prodotto estetizzante, profondamente intellettuale e anticommerciale. L'aspetto provocatore del cineasta, sempre sulla breccia della lotta personale che porta avanti contro la società borghese e la cultura di massa, appare qui con grande chiarezza: Pasolini, a cui non piace l'opera, giacché la considera il simbolo della cultura borghese per eccellenza ${ }^{23}$, per il suo film si serve giustamente dell'icona operistica più prestigiosa ancora a quell'epoca, la quale, anche per le sue origine elleniche modeste, finisce col diventare una sorta di mito al quadrato da cui è egli stesso affascinato, come afferma nell'intervista rilasciata a Jean Duflot nel 1969:

Ho pensato subito a Medea sapendo che il personaggio sarebbe stato lei. Delle volte scrivo la sceneggiatura senza sapere chi sarà l'attore. In questo caso sapevo che sarebbe stata lei, e quindi ho sempre calibrato la mia sceneggiatura in funzione della Callas. [...] Cioè, questa barbarie che è sprofondata dentro di lei, che viene fuori nei suoi occhi, nei suoi lineamenti, ma non si manifesta direttamente, anzi, la superficie è quasi levigata, insomma i dieci anni passati a Corinto, sarebbero un po' la vita della Callas. Lei viene fuori da un mondo contadino, greco agrario, e

17. Il 30 marzo 1960, alla Clinica Dezza (Via Dezza 48) di Milano, alle 8 del mattino, Maria Callas aveva subito un parto cesareo dando alla luce un maschietto prematuro di otto mesi. A causa di un'insufficienza respiratoria, il neonato era stato trasportato in un'altra clinica dotata di apparecchiature più moderne ed era morto nell'ambulanza durante il tragitto dopo essere stato battezzato, con nome di Omero Lentini, dall'infermiera che l'accudiva. La morte del neonato, le spiegò il dottor Carlo Palmieri, il suo ginecologo, era dovuta allo sviluppo insufficiente dei polmoni. Tuttavia, era stata lei, in preda a una crisi di panico dovuta all'assenza prolungata di Onassis, ad aver supplicato il dottor Palmieri e ad averlo convinto a farla partorire col cesareo non appena sarebbe stato possibile (Cf. GAGE, N. op . cit., p. 235). La Callas era incinta di Onassis dalla prima crociera sullo yacht Christina svoltasi dalla fine del luglio 1959 e non si era più mostrata in pubblico dal dicembre dello stesso anno, eccezion fatta per l'intervista concessa nel febbraio seguente a Marylise Schaeffer, 
poi si è educata per una civiltà borghese. Quindi in un certo senso ho cercato di concentrare nel suo personaggio quello che è lei, nella sua totalità complessa ${ }^{24}$.

Il film di Pasolini vuole essere un'elaborazione poetica sul mito di Medea: si tratta, infatti, dell'ultima parte del filone "classico" del cineasta, l'ultimo lavoro della trilogia inaugurata con Il Vangelo secondo Matteo (1964) e Edipo Re (1967) in cui domina il conflitto tra il sacro e il profano. La trama del film segue fedelmente il mito di Medea: inizia dall'infanzia di Giasone, educato dal centauro Chirone, prosegue poi con il viaggio degli Argonauti alla ricerca del vello d'or, con l'aiuto che la maga fornisce a Giasone dopo aver fatto a pezzi suo fratello Apsirto, continua con l'arrivo a Corinto e con la loro vita comune felice di alcuni anni con i loro due figli, giunge al ripudio di Medea a opera di Giasone, al matrimonio di quest'ultimo con Glauce, la figlia del re di Corinto e, infine, alla morte di costei (e di suo padre) nonché all'infanticidio per mano di Medea.

Al centro di quest'opera cinematografica vi è l'idea della perdita del sacro con, sono i termini utilizzati da Pasolini, la "conversione a ritroso" di Medea, il che la conduce direttamente alla propria fine dopo aver provato un certo sollievo, per qualche tempo, grazie alla passione carnale di Giasone. Volendo schematizzare al massimo, si potrebbe dire che Medea e il suo mondo sono la metafora della pre-civiltà, del caos, della barbarie, cioè dell'eden stando a Pasolini, luogo spazio-temporale in cui domina l'idea del sacro, ove tutto è regolato da un tempo ciclico e dove il dionisiaco e l'irrazionale hanno libero corso. Il cineasta ci presenta questo mondo sotto l'aspetto di una Colchide reinventata con i suoi paesaggi aridi e dirupati e le sue grotte trovate e filmate in Cappadocia. Tale mondo primordiale rappresenta ai suoi occhi l'era della Natura, resa simbolicamente anche attraverso l'arte rupestre e i sacrifici umani in onore degli dei.

Giasone e il suo mondo sarebbero invece una metafora della Grecia, vale a dire della modernità, della civiltà, dell'ordine e del razionale, ma anche dei nuovi rapporti umani, profani, disinvolti, pragmatici: il regno del tempo lineare, del profitto che avanza, dunque del mondo capitalistico secondo l'ideologia pasoliniana, dell'apollineo. Nel suo film Pasolini reinventa quindi la città di Corinto dandole le linee geometriche

al Ritz, per France-Soir, pubblicata il 13 febbraio 1960 col titolo «La Callas m'a dit: je n'ai plus envie de chanter... Je voudrais un enfant» ("La Callas mi ha detto: non ho più voglia di cantare.. Vorrei un figlio"). Nell'intervista, durata quasi due ore, l'artista non aveva fatto nascere alcun sospetto nella giornalista. Nel 1968, invece, mentre Onassis flirta ormai con Jackie Kennedy, la Callas metterà in giro la voce che era stato lui a farla abortire.

18. «À propos de Médée», intervista con Laura Betti, trasmessa sul canale televisivo ARTE nel settembre 1997, (C) La Sept ARTE FIT PRODUCTION SODAPERAGA, Francia, 1997.

19. Il pittore Giuseppe Zigaina, amico fraterno di Pasolini e suo collaboratore per vari film, tra cui Medea, ha partecipato alla riprese 
perfette della piazza dei Miracoli di Pisa, espressione grandiosa dell'era della Cultura. L'incontro di questi due mondi produce l'effetto di uno shock di culture e ciò va risituato all'epoca in cui vive e lavora il cineasta, la fine degli anni 1960, in una società occidentale in piena trasformazione, il che è anche un leitmotiv della sua filmografia, in cui il mondo del proletariato e del pre-industriale si scontra con quello industrializzato, capitalista, urbano e benpensante dell'Italia di quegli anni. Un divario profondo tra due diverse concezioni del mondo e della società e anche fra due "Italie" ${ }^{25}$. In effetti Pasolini dichiara che nella sua Medea non si tratta di una trasposizione della tragedia di Euripide, ma che "l'intero dramma poggia su questa contrapposizione di due 'culture', sull'irriducibilità reciproca di due civiltà. [...] potrebbe essere benissimo la storia di un popolo del Terzo Mondo, di un popolo africano, ad esempio, che vivesse la stessa catastrofe venendo a contatto con la civiltà occidentale materialistica" ${ }^{26}$. La tragedia sgorga allora dalla nascita del mondo moderno, un mondo senz'anima in cui non vigono più il senso del sacro e dei veri valori ("Del resto, nell'irreligiosità, nell'assenza di ogni metafisica, Giasone giunge al punto di essere lui il nesso con la nostra storia moderna"). Medea è una sorta di inno al paradiso perduto, filmato, in particolare, proprio nelle prime sequenze, durante le scene sacrificali e del cannibalismo, in quanto si tratta di sacrificio vitale, pregno di spiritualità, che nutre la terra e il sole, segni che rinviano ai genitori di Medea. La "conversione a ritroso" (vedi la poesia "Endoxa", p. 1893) della maga e la sua follia omicida sono in fin dei conti dovute allo sradicamento, alla perdita delle proprie origini e dei propri valori, alla perdita del sacro. Ecco quanto afferma Pasolini in un'intervista:

Medea [...] è giunta al massimo dell'integrazione; qual è mai la causa profonda di questa autoesclusione in Medea? Una specie di conversione a ritroso. Immagini che san Paolo fosse credente nel momento in cui precipitò da cavallo, e il trauma gli avesse fatto perdere la fede. Medea è vittima della stessa "folgorazione". Non che Giasone l'abbia convinto di inseguirlo, ma lo fa al di fuori dell'intervento di qualsiasi ragionamento logico. A un certo punto ha una visione di Giasone, e scatta l'irreparabile ${ }^{27}$.

\footnotetext{
del film e ha affermato: "Maria si era proprio follemente innamorata di Pier Paolo e sognava di sposarlo. Me lo confidò lei stessa, tra le lacrime, verso la fine delle riprese del film, chiedendomi di aiutarla a raggiunger il suo scopo. Mi trovai in una situazione molto complicata perché Pier Paolo era notoriamente omosessuale, e non fu facile per me agire con delicatezza, senza ferire la sensibilità di nessuno dei due" (Allegri, R. e R. Callas by Callas, Milano, Mondadori, 1997, p. 148).

20. Ibidem.

21. Ibid.

22. "C'è un mistero Callas, ci sono degli enigmi che sono indipendenti dalla carriera e che appartengono precisamente al mito" (Dictionnaire des mythes d'aujourd'hui, BRUNEL, P. (dir.), Paris, édition du Rocher, 1999, p. 144).
} 
Ella uccide perché non accetta la sua nuova condizione che è quella della Storia che avanza inesorabilmente; tuttavia, uccidendo, ella commette paradossalmente un atto d'amore dato che questo gesto sottrae i propri figli al tempo lineare, perituro, umano, iscrivendoli così in un tempo mitico, come sottolinea giustamente Christophe Mileschi: "riconciliata con la propria fede e con i propri poteri di un tempo, ella li riporta così, sul carro del dio Sole, padre di suo padre, nel tempo litico e sacro, nel tempo cosmico in cui amarli, nell'infinito carosello delle resurrezioni'”28. Pasolini non manca inoltre di mettere in scena se stesso attraverso la figura, duplice, del centauro-uomo, Chirone, il suo portavoce ${ }^{29}$. L'idea del doppio e di una dialettica binaria è d'altronde presente a vari livelli nella diegesi filmica. Agli occhi di Giasone, Chirone appare sotto forma di centauro e di uomo, di essere mitico e sacro - divino - e umano e profano - terrestre; lo spettatore assiste a una duplice versione del sacrificio umano: rituale e cannibalesco all'inizio del film, quando è prerogativa del popolo, razionalmente meditato e teatralizzato quando Medea immola i propri figli ${ }^{30}$. C'è anche, tuttavia, una duplice versione - ancora una volta sacra e profana - della morte di Glauce e di suo padre. Mentre gira questo film "letterario", il cineasta realizza una serie di disegni e scrive, soprattutto, le "Visioni di Medea": si tratta di 98 testi contenenti, oltre le scene già realizzate, degli abbozzi di riflessioni poetiche e filosofiche sul sacro e il profano, che pubblica all'uscita del film con i dialoghi definitivi e con venticinque poesie scritte durante le riprese ${ }^{31}$.

La desacralizzazione di un'epoca di cui parla Medea di Pasolini trova, inoltre, un'eco nella demitizzazione della Callas, di questo mito moderno: da cantante "divina", era questo il suo epiteto, abituata al successo tributatole dalle folle in quanto diva, ora, come donna in carne ed ossa e priva del suo "magico" attributo, la "voce", si ritrova destinata in fin dei conti, scientemente, grazie al lavoro del cineasta, a un pubblico di nicchia. Ciononostante, come ha giustamente osservato la critica, quello della Callas, anche se muta, resta un corpo "necessariamente operistico, votato al dramma, al grido e al furore" 32 .

Le recensioni del film furono buone, ma il pubblico rimase freddo: malgrado un successo di stima per l'attrice ${ }^{33}$, Medea di Pasolini non incassò e nemmeno lanciò la

\footnotetext{
23. Tengo a sottolineare, però, che Pasolini prende in considerazione e attacca soltanto l'aspetto mondano più visibile (e forse più ridicolo), quello dei diamanti, delle pellicce e dei ricevimenti lussuosi del jet set al quale è oramai ridotta l'opera in un'Italia in crisi alla fine degli anni '60, negando in tal modo a tale genere artistico, italiano per antonomasia, il suo carattere "popolare" nel senso nobile del termine.

24. Duflot, J. (org.), Pier Paolo Pasolini. Il sogno del centauro, Roma, Editori Riuniti, 1983, in <http://www.pasolini.net/10tesimy_capitoloterzo03.htm >

25. Cf. Mileschi, C. «Présentation », in: Pasolini, P. P. Médée, op. cit., p. 7.

26. Pasolini, P. PIl sogno del centauro, (titolo originale: Les dernières paroles d'un impie, trad. di Martine Schruoffeneger, Pierre Belfond, 1981), a c. di Jean Duflot, introd. di Gian Carlo Ferretti, Roma, Editori Riuniti, (1983) 1993, p. 103.
} 
carriera cinematografica della Callas che, di fatto, non riapparirà più sul grande schermo.

\section{Per concludere}

Maria Callas non è una cantante che alcuni ammiratori hanno semplicemente mitizzato, ma incarna un mito giacché i meccanismi del mito coincidono con la vita e le tracce da lei lasciate. La Callas continua a essere un vettore che si muove nel tempo e nell'immaginario collettivo per partecipare in modo decisivo all'edificazione di un pilastro della nostra civiltà: la questione della condizione umana ${ }^{34}$. Eccoci allora tornare, in ultimo, alla tragedia di Medea: la maga della Colchide, nipote del Sole e di Circe, affetta da un complesso di mascolinità, come direbbero le teorie psicanalitiche, accettando la propria natura umana di donna, di sposa e di madre, sprofonda in una crisi che, come ricorda Mathilde Landrain, è di "di natura religiosa" 35 . È giocoforza allora costatare che questo mito è stato spesso rielaborato e riproposto in epoche di crisi: già in Euripide, poi negli anni della Rivoluzione francese da Cherubini, indi alla fine degli anni 1960 da Pasolini, in un'Italia profondamente straziata tra cattolicesimo in dissesto, capitalismo nascente e inizio del terrorismo e, infine, negli ultimissimi anni del secolo scorso, il $20^{\circ}$, e nei primissimi dell' attuale, periodo costellato di guerre e di ritorni a forti valori religiosi.

Perché l'interesse per tale mito sembra riapparire, rinnovarsi e associarsi a epoche storiche così precise? Medea non sarebbe forse, in fin dei conti, l'espressione condensata e altamente simbolica della crisi del presente e della visione pessimistica del futuro, in particolare con la distruzione della prole? Ma anche, dopotutto, un modo di esorcizzare la crisi di valori che attraversa ogni società in momenti chiave difficili della propria storia grazie alla proiezione dei propri fantasmi che, come nel caso di Medea, mostrano, in guisa di avvertimento, tutta la crudeltà di cui l'uomo e la società umana sono capaci.

27. Ibidem.

28. Mileschi, C. op. cit., p. 10.

29. Cf. Pasolini, P. P. Medea, 2 DVD 786933, nuovo Master restaurato, (1969) 2004, Carlotta Films / CIPA / Allerton Films). Per maggiori spunti sul film, in particolare sulla dialettica verbale-non verbale e sull'uso delle musiche, $c f$. l'ottimo sito <http://www.pasolini.net/8tesimy_capitoloterzo01.htm>.

30. Come si fa a non pensare qui alla vita privata della Callas, all'episodio della morte di suo figlio di cui ha dovuto sentirsi responsabile e al senso di colpa che ha forse ritrovato interpretando il ruolo di Medea per il grande schermo? 
31. Poesia "Callas", in Poesie disperse I: "Tutto cessa da sé, quando la (il) cliente / non ha più soldi”, disse il collega cinico / a Jung. Ma niente è mai cominciato! C'è stato / forse il momento inaugurale in cui la vittima / da buttare nel fuoco fu sostituita (presso gli Scozzesi) / da un fantoccio? / Le due cose furono (e sono) sempre contemporanee. / I superamenti, le sintesi! sono illusioni, / dico io, da volgare europeo, ma non per cinismo - / e Lévy-Bruhl fondava il razionalismo delle società... / superiori (e lo faceva a ragione) su "tempo, spazio e sostanza", / terna di cui mancano (e avevano dunque ragione... / le società inferiori) i due primi dati! La tesi / e l'antitesi convivono con la sintesi: ecco / la vera trinità dell'uomo né prelogico né logico, / ma reale. Sii, sii scienziato con le tue sintesi / che ti fanno procedere (e progredire) nel tempo (che non c'è), / ma sii anche mistico curando democraticamente / nel medesimo tabernacolo, con sintesi, tesi e antitesi. / La storia non c'è, diciamo, c'è la sostanza: che è apparizione. / Così potrai vivere, da bravo cittadino rivoluzionario, / nella storia che è la sola illusione possibile per te. / Infatti, il sole domenicale splende, e fervono lavori / di falegnameria, evidentemente supplementari o "straordinari", / nella pace del sole della Cappadocia, la cui realtà / dopo una lunga interruzione, riprende il suo corso, / attendendo, oggi, ad esempio, l'arrivo della Callas. / (Sotto questo sole, poi, sia detto ancora, per incidente, San Paolo è passato invano. Migliaia di creature / hanno continuato senza interruzione e pace / a pascolare greggi se maschi, a zappare e filare se femmine; / tutti ugualmente soffocati da polvere o fango secco - / mentre supremamente indifferenti mandorli e pistacchi / convivono smaglianti / con chi ha ancora un po' di soldi da spendere.) giugno 1969 (in P. P. PAsolini, Bestemmia. Tutte le poesie, a c. di Graziella Chiarcossi e Walter Siti, pref. di Giovanni Giudici, Milano, Garzanti, 1993, II, p. 1903-1904)

32. Cf. «Médée, un film de Pier Paolo Pasolini», Une chronique de John Constantine, in http://www.dvdclassik.com/Critiques/medee-dvd.htm (gennaio 2005). Va aggiunto che si assiste qui anche alla desacralizzazione del mitico viaggio degli Argonauti che viene praticamente ridotto a un unico piano fisso riproducente la "Zattera della Medusa" di Delacroix.

33. Alla domanda su come abbia fatto a convincere la Callas ad accettare il ruolo di Medea, Pasolini risponde: "Ciò che forse non si sa è che avevo già pensato alla Callas per la parte di Giocasta in Edipo re... Fatto sta che impersonava per me, da tempo, una serie di figure femminili del repertorio tragico... Sono riuscito a convincerla grazie alla mediazione del produttore Rossellini, amico personale della Callas. Sapevo che non avrebbe mai accettato di realizzare per lo schermo la sua interpretazione di Medea nell'opera lirica di Cherubini. Devo dire che è un'attrice nata, di un'intelligenza spontanea e di una presenza del tutto eccezionali... Una delle attrici che mi hanno posto meno problemi di direzione" (Il sogno del centauro, op. cit., p. 113).

34. Dictionnaire des mythes d'aujourd'hui, op. cit., p. 152.

35. "La tragedia greca sarebbe apparsa in modo miracoloso nel VI secolo a.C. derivata, secondo Aristotele, dal ditirambo [...]. La sua matrice è di natura religiosa [...]" (LANDRAIN, M. op. cit., p. 5). 